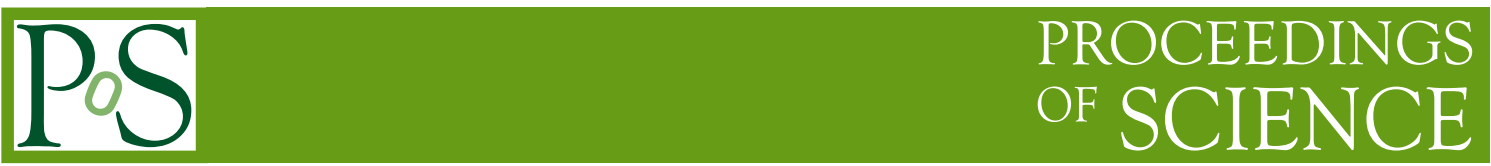

\title{
A simulation framework for spherical proportional counters
}

loannis Katsioulas, ${ }^{a}$ Patrick Knights, ${ }^{a}$ Jack Matthews, ${ }^{a}$ Thomas Neep, ${ }^{a, *}$ Konstantinos Nikolopoulos ${ }^{a}$ and Robert James Ward ${ }^{a}$

${ }^{a}$ University of Birmingham

E-mail: t.j.neep@bham.ac.uk

Spherical proportional counters are novel gaseous detectors with numerous applications, including neutron spectroscopy and searches for dark matter and neutrinoless double beta decay. A simulation framework is presented that combines the Geant 4 and Garfield++ toolkits. Example results from the application of this simulation framework in different experimental contexts are shown.

40th International Conference on High Energy physics - ICHEP2020

July 28 - August 6, 2020

Prague, Czech Republic (virtual meeting)

${ }^{*}$ Speaker 


\section{Introduction}

Spherical proportional counters [1] (SPCs), are novel gaseous detectors with multiple applications including searching for low-mass dark matter [2], neutrinoless double beta decay measurements $[3,4]$ and neutron spectroscopy $[5,6]$. A simulation framework has been developed to study SPCs [7] by combining the Geant4 [8] and Garfield++ [9] toolkits. Geant4 is a toolkit for simulating the passage of particles through matter. Garfield++ is a toolkit dedicated to the simulation of gaseous detectors, interfacing several programs such as Magboltz [10] for simulation of electron transport in gases, programs that implement the finite element method (FEM), such as Ansys [11], for modelling electric fields, and Heed [12] to simulate the ionisation patterns produced by relativistic charges particles. The combination of these programs allows the characteristics of SPCs with various configurations (detector geometries, materials, and gases) to be studied in detail, which is crucial for both detector design and interpretation of measured data.

\section{Spherical proportional counters}

SPCs consist of a spherical grounded metallic shell, which acts as a cathode, containing a gas volume and central anode sensor. The anode is kept at a high voltage and supported by a grounded metallic rod, producing a radial electric field. The small detector capacitance, nearly independent to the detector radius, allows the construction of large sensitive volumes, while the optimal volume-to-surface ratio is well suited to low background applications. In addition, the target gas can be easily adapted to the use case. Figure 1 shows a schematic of an SPC, showing the major components of the detector.

As ionising particles travel through the gas volume they produce electrons in the gas. In the influence of the electric field these electrons drift towards the anode at the centre of the detector where the electric field is strong enough to cause them to avalanche, producing a large

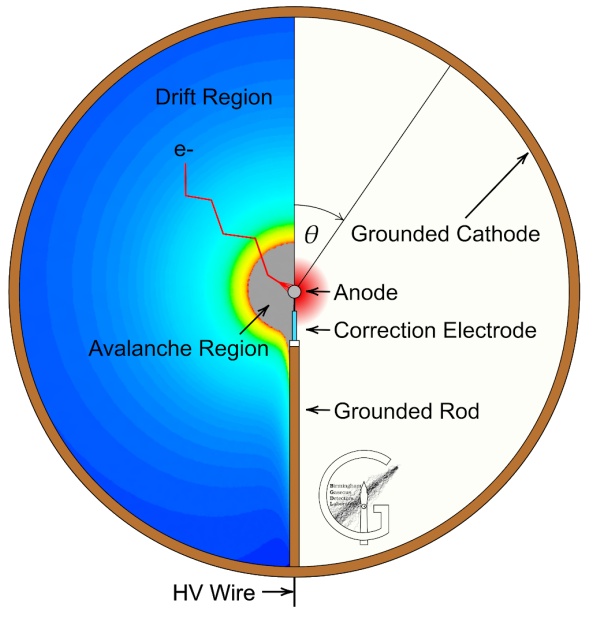

Figure 1: Operational principle of the spherical proportional counter number of electron-ion pairs. The produced electrons (ions) then drift towards (away from) the anode, inducing a current signal which is read out by a data acquisition system.

\section{Simulation outline}

The simulation framework brings together two software toolkits, Geant 4 and Garfield++, to simulate the complete process of an interaction in an SPC. A demonstration of the combination of these toolkits was presented in Ref [13]. To begin, the detector is described in Geant 4 and the G4ParticleGun class is used to produce the primary particle, which offers a flexible choice of particle type, starting position, direction and energy. In addition to generating "simple" particles, such as electrons and photons, the decay of radioactive isotopes is possible through the use of the G4RadioActiveDecay model. 
Geant4 is used to model the interaction of particles inside the detector until electrons are produced with a kinetic energy, $E<2 \mathrm{keV}$. At this point a user model is triggered and the electrons are passed to the Garfield++ part of the simulation. Initially, Heed is used to produce $\delta$-electrons, before electrons are drifted towards the anode using the AvalancheMC class of Garfield++, until they arrive in the "avalanche region", where the electric field is strong. The electron drift relies on gas files and electric field maps produced by Magboltz and Ansys, respectively.

In the avalanche region, the average gain of each electron is calculated by numerically integrating the Townsend coefficient minus attachment coefficient along the electrons path, to estimate the number of electron-ion pairs produced in the avalanche. The ions created in this step are then drifted away from the anode and towards the cathode, inducing a current signal. This current signal is then convolved with the response of the read-out electronics, producing a voltage pulse like those measured in real detectors.

Each voltage pulse can be analysed to extract information about the kind of interaction occurred. Properties such as the integral and maximum amplitude of the pulse provide information about how much energy was deposited in the event, while temporal features such as the risetime (the time it takes for the pulse to go from $10 \%$ to $90 \%$ of its maximum amplitude), can be used to discriminate track lengths. Typically two or more of these variables are used simultaneously in an analysis to discriminate between different kinds of interaction.

\section{Applications of the simulation framework}

In this section a number of different applications of the simulation framework are demonstrated.

\subsection{Neutron spectroscopy}

It has been proposed that SPCs can be used as neutron spectrometers when filled with nitrogen gas, $N_{2}$ [5]. In this setup, interactions of neutrons with nitrogen occur through two main processes

$$
\begin{aligned}
& { }^{14} \mathrm{~N}+n \rightarrow{ }^{14} \mathrm{C}+p+625 \mathrm{keV} \\
& { }^{14} \mathrm{~N}+n \rightarrow{ }^{11} \mathrm{~B}+\alpha-159 \mathrm{keV}
\end{aligned}
$$

In the simulation, it is possible to determine which interaction took place. Figure 2 shows simulation results from neutrons interacting with an SPC filled with $\mathrm{N}_{2}$. Figure 2a shows multiple events overlayed, showing the tracks left by process 1 (magenta), process 2 (teal) and by elastic scattering (orange). It can be seen that the tracks left by protons (magenta) are longer than the tracks left by $\alpha$ particles (teal). The length of the proton tracks means that many of them hit the cathode of the detector before depositing all of their energy, while the majority of the $\alpha$ particle events are fully contained. These different properties, that can be used for particle identification, manifest in the measured pulses shown in Figure $2 \mathrm{~b}$ and a separation between the three interaction types is visible in the two-dimensional distribution of pulse integral versus risetime in Figure 2c.

\subsection{Neutrinoless double beta decay searches with R2D2}

R2D2 (Rare Decays with a Radial Detector) [3] aims to use SPCs in the search for neutrinoless double beta decay $(0 v \beta \beta)$. Such a search requires excellent energy resolution of order $1 \%$ full-width 


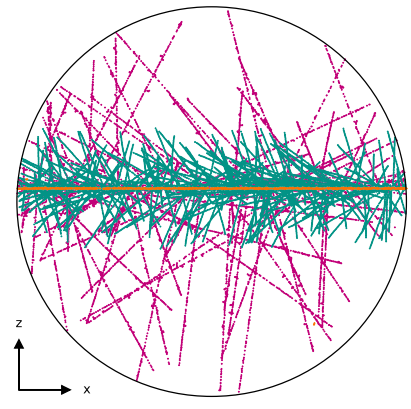

(a)

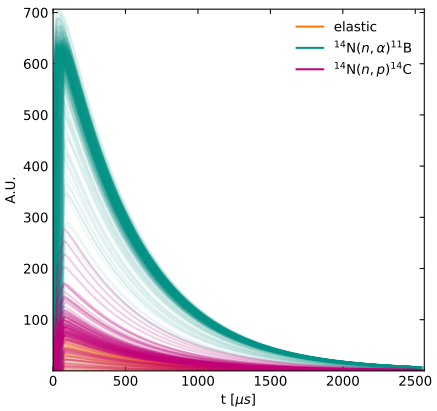

(b)

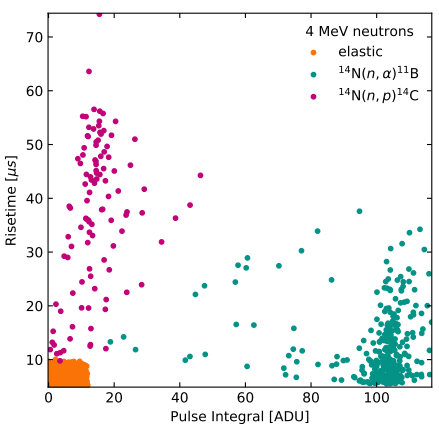

(c)

Figure 2: Simulated (a) tracks, (b) pulses and (c) pulse integral versus risetime distributions of $4 \mathrm{MeV}$ neutrons interacting with $\mathrm{N}_{2}$ in an SPC

at half-maximum (FWHM). In order to study this, the collaboration has made measurements of the energy resolution of $\alpha$ particles from a ${ }^{210} \mathrm{Po}$ source in a $20 \mathrm{~cm}$ diameter SPC filled with a gas composed of $99 \% \mathrm{Ar}$ and $2 \% \mathrm{CH}_{4}$ at 200 mbar [14].

The simulation framework has been used to complement these measurements. Figure 3 shows a comparison between data and simulation of Qt, the integral of the current pulse, versus Dt, the time the current pulse is greater than a threshold of $1 \%$ of the maximum amplitude. It can be seen that the two distributions show good agreement. Additional information available only in the simulation can be used to determine the angle that the $\alpha$ particles were emitted at, represented by the colour of each data point in the plot from the simulation.

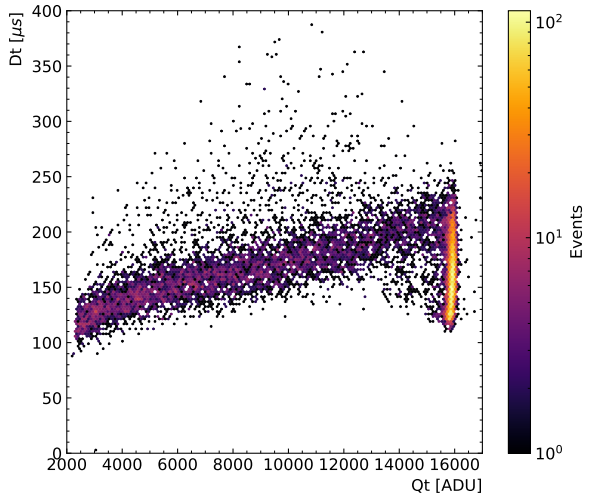

(a)

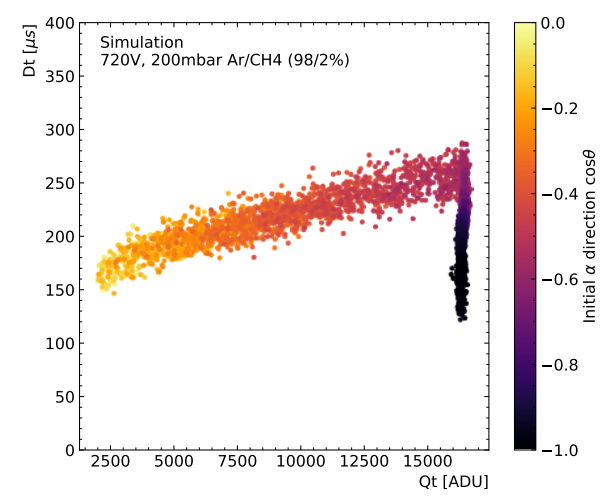

(b)

Figure 3: (a) Measured and (b) simulated current integral (Qt) versus time over threshold (Dt) distributions of 5.3 MeV $\alpha$ particles from a 210 Po source in a $20 \mathrm{~cm}$ diameter SPC filled with $98 \% \mathrm{Ar}$ and $2 \% \mathrm{CH}_{4}[14]$

\subsection{Multi-anode sensor (ACHINOS)}

The NEWS-G collaboration [2] is searching for light dark matter using an SPC. The collaborations latest detector, SNOGLOBE, is $135 \mathrm{~cm}$ in diameter and uses a recently developed, multi-anode sensor known as ACHINOS $[15,16]$. The ACHINOS sensor offers several advantages over a traditional single-anode sensor [17]. The design of the sensor means that stronger electric fields can be 
produced in the volume of the detector independently of the gain in the avalanche region, which is particularly important for operating in large detectors and/or high pressures. Another advantage is that individual anodes, or groups of anodes, can be read out separately, allowing for segmentation of the detector which can be used to determine where in the gas volume an interaction occurred.

A simulation of an eleven-anode ACHINOS sensor with two read-

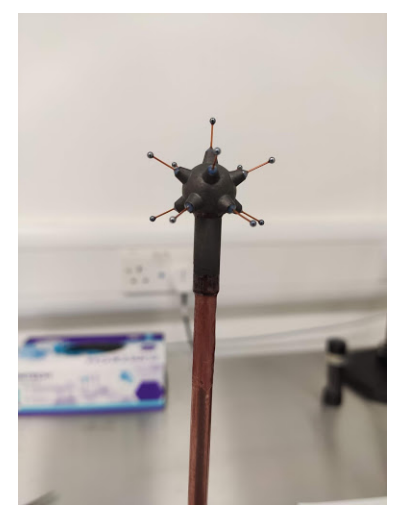

Figure 4: Realisation of an ACHINOS sensor out channels has been performed. A photograph of such a sensor is shown in Figure 4 showing the orientation of the anodes. Figure 5, shows the operating electric field and the two weighting fields produced by the sensor for the six anodes on the side of the sensor away from the supporting rod. It can be seen that the far-from-rod weighting field lines (red) follow the electric field lines (blue), while the near-rod weighting field lines (green) are in the opposite direction in some cases. The impact of this is that when an ion drifts away from one of these anodes it will induce a positive signal in one readout along with a negative signal in the other due to Ramo-Shockley theorem. Figure 6a shows a simulated pulse for an electron produced near the supporting rod. It can be seen that the large pulse from the rod-side readout is accompanied by a small negative pulse from the far-side readout. The risetime of each pulse related to the radius of the interaction, as shown in Figure 6b.

The two readout channels offer the potential to distinguish where in the detector in initial interaction occurred by constructing an asymmetry of the near- $(\mathrm{N})$ and far-pulse $(\mathrm{F})$ amplitudes, $A=\frac{F-N}{F+N}$. Figure $6 \mathrm{c}$ shows the value of $A$ in different regions of a detector, demonstrating this.
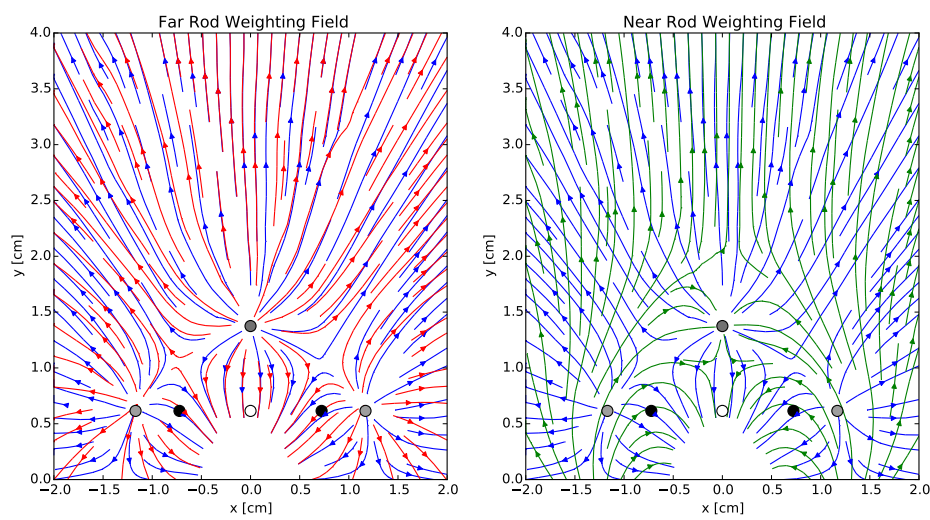

Figure 5: Weighting (red/green) and electric field lines (blue) for a two-channel eleven-anode ACHINOS

\section{Summary}

A simulation framework for spherical proportional counters has been developed. The framework combines the Geant 4 and Garfield++ toolkits to produce a complete detector simulation.

Several use cases of the simulation framework have been demonstrated, including comparisons of the simulation results with data from the R2D2 experiment, which demonstrate good agreement. Work is ongoing in the context of several different experiments to further validate the simulation. 


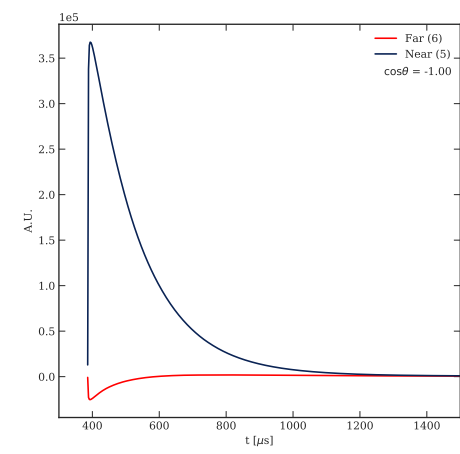

(a)

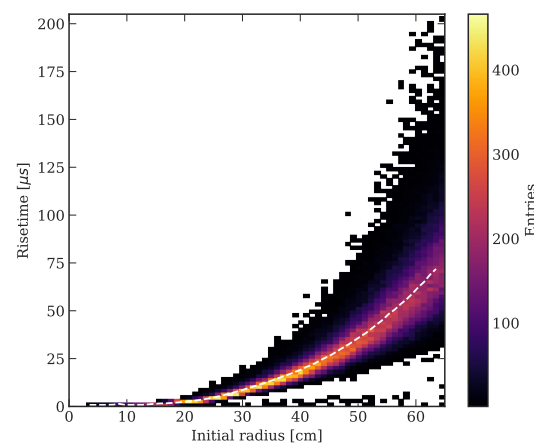

(b)

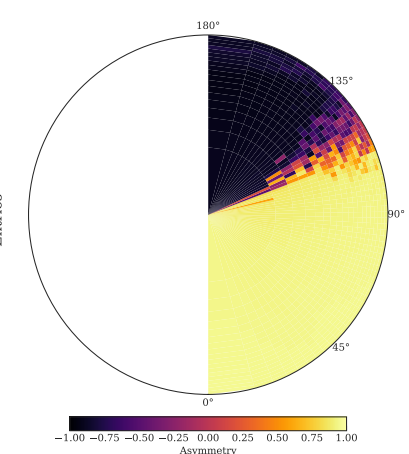

(c)

Figure 6: (a) Simulated pulses, (b) risetime versus radius, and (c) asymmetry distribution of electrons simulated in an SPC with a two read-out channel ACHINOS sensor

\section{References}

[1] I. Giomataris et al., A novel large-volume spherical detector with proportional amplification read-out, Journal of Instrumentation 3 (2008) 7.

[2] NEWS-G collaboration, First results from the NEWS-G direct dark matter search experiment at the LSM, Astropart. Phys. 97 (2018) 54 [1706.04934].

[3] A. Meregaglia et al., Study of a spherical Xenon gas TPC for neutrinoless double beta detection, JINST 13 (2018) P01009 [1710.04536].

[4] C. Jollet, A new neutrinoless double beta decay experiment: R2D2, J. Phys. Conf. Ser. 1468 (2020) 012108.

[5] E. Bougamont et al., Neutron spectroscopy with the spherical proportional counter based on nitrogen gas, Nucl. Inst. Meth. A 847 (2017) 10 .

[6] I. Katsioulas et al., Fast Neutron Spectroscopy with a Nitrogen-Based Gaseous Detector, in 2019 IEEE Nuclear Science Symposium (NSS) and Medical Imaging Conference (MIC), pp. 1-3, 2019, DOI.

[7] I. Katsioulas et al., Development of a simulation framework for spherical proportional counters, JINST 15 (2020) C06013 [2002 .02718].

[8] S. Agostinelli et al., Geant4—a simulation toolkit, Nucl. Inst. Meth. A 506 (2003) 250 .

[9] R. Veenhof, GARFIELD, recent developments, Nucl. Instrum. Meth. A 419 (1998) 726.

[10] S.F. Biagi, Magboltz 11, http://magboltz.web.cern.ch/magboltz.

[11] Ansys ${ }^{\circledR}$, release 19.1, https://www.ansys.com/academic.

[12] I. Smirnov, Modeling of ionization produced by fast charged particles in gases, Nucl. Instrum. Meth. A 554 (2005) 474.

[13] D. Pfeiffer et al., Interfacing Geant4, Garfield++ and Degrad for the Simulation of Gaseous Detectors, Nucl. Instrum. Meth. A 935 (2019) 121 [1806.05880].

[14] R. Bouet et al., R2D2 spherical TPC: first energy resolution results, 2007.02570.

[15] A. Giganon et al., A multiball read-out for the spherical proportional counter, JINST 12 (2017) P12031 [1707.09254].

[16] I. Giomataris et al., A resistive ACHINOS multi-anode structure with DLC coating for spherical proportional counters, 2003.01068.

[17] I. Katsioulas, I. Giomataris, P. Knights, M. Gros, X. Navick, K. Nikolopoulos et al., A sparkless resistive glass correction electrode for the spherical proportional counter, JINST 13 (2018) P11006 [1809. 03270]. 